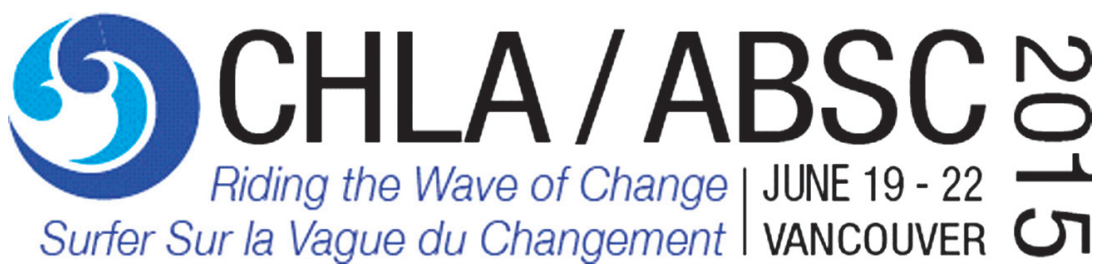

Canadian Health Libraries Association 2015 Conference

19-22 June 2015

Vancouver, British Columbia, Canada
Association des bibliothèques de la santé au Canada Congrès 2015

19 au 22 juin 2015

Vancouver, British Columbia, Canada

\title{
Contributed papers / Communications libres
}





\title{
CONTRIBUTED PAPERS / COMMUNICATIONS LIBRES
}

\section{Creative and Imaginative Searching: Health Science Librarians' Strategies and Barriers to Providing Good Service}

\author{
Jill McTavish \\ Clinical Librarian, London Health Sciences Centre, London, ON
}

Background: Proponents of evidence-informed medicine (EIM) from the 'caring' professions, such as nursing and social work, are increasingly critiquing narrow interpretations of evidence-based practice (EBP). When health science librarians (HSLs) are providing information to users-especially users from these disciplines-they must be aware of these critiques and how they apply to their services. In this project I explore HSLs' strategies for providing good service, especially mediated literature searching service, to nursing and allied health professionals. Methods: Twenty-two HSLs from across Canada who provide service for nurses and allied health professionals were recruited through professional associations and listservs that represent this group. Participants completed a $30 \mathrm{~min}$ telephone interview about perceived barriers and strategies to providing 'good service' at their libraries and also responded to three challenging search scenarios. Interviews were transcribed and thematic analysis was performed. Results: The findings indicate that HSLs across Canada have divergent searching practices and strategies for dealing with complex search queries. For example, some HSLs only complete scoping reviews while others have developed non-linear strategies for answering queries that appear to be unsupported by evidence. Discussion: Proponents of EIM value practitioners as creative and imaginative agents, rather than as mechanical practitioners of EBP. HSLs' nuanced strategies for answering complex search queries also allude to their creative and imaginative search practices. These findings underscore the limitations of one size fits all literature searching service and suggest a need for an advanced understanding and application of EBP in health science librarianship.

\section{Everyday Life Health Information Seeking Research: Mapping the Literature}

\author{
Devon Greyson \\ Information Specialist and Doctoral Candidate, University of British Columbia, Vancouver, BC
}

Introduction: Following the wave of changes to health information access brought by the consumer health movement and the development of the World Wide Web, librarians and information scientists have increasingly recognized the importance of everyday life information seeking (ELIS). The objective of this paper is to map and assess how health ELIS has been studied in the (English, Spanish and French) library and information science literature, including the extent to which it addresses priority health issues. Methods: To map the health ELIS literature, identify trends and themes therein, and assess the use of theories, methods, and sources of evidence, a scoping study was conducted. Results: From a total of 424 initially identified citations, 89 articles (published 20002014) met study inclusion criteria. Fifty-one percent employed quantitative methods, $24 \%$ qualitative, $16 \%$ mixed-methods, and 9\% were reviews. The majority of studies focused on adults, and it appears that women may be overrepresented. Authors and study populations were highly concentrated in a few wealthy countries. Half of the articles focused on no specific health topic; beyond that 23 different topics were studied, few of which correspond with

Please note that all presentation slides have been uploaded to the ABSC/CHLA conference website and can be accessed online here:

http://chla-absc.ca/conference/program/sessions/archive. 
health priority issues. Use of theory and conceptual models was inconsistent, and only a minority attempted to incorporate concepts of context. Discussion: Few aspects of the health ELIS literature align with current public health priorities. While in some cases this may be appropriate, looking at the literature as a whole, one can conclude that more health ELIS research should centre on priority health issues and populations.

\title{
The Need for Standards for Literature Searching in Health Libraries
}

\author{
Brooke Ballantyne Scott, Susan Baer, Marcus Vaska, Jackie MacDonald, \\ Lori W. Léger, Ashley Farrell, and Pat Lee
}

Librarian, Royal Columbian Hospital, New Westminster, BC; Director of Libraries \& Archives, Regina Qu'Appelle Health Region, Regina, SK; Librarian, Knowledge Resource Service, Holy Cross Centre, Alberta Health Services, Calgary, AB; Privacy Officer, Annapolis Valley Health and South Shore Health, Kentville \& Bridgewater, NS; Regional Manager, Horizon Health Network, Fredericton, NB; Research Associate, Cancer Care Ontario, Toronto, ON; Retired Health Librarian, Halifax, NS

Introduction: Seeing the need for shared health services library standards for consistency within our own multi-site services, for teaching health librarianship, and for quality improvement indicator development, a group of Canadian librarians formed a "standards working group". The main goal of this group was to develop a set of standards for literature searching in health services libraries across Canada. Methods: In the summer of 2014, we started work on a literature searching standard for health services libraries written by librarians, for librarians. We are interested in creating a standard that encompasses the various types of day-to-day searches completed by health librarians, rather than a standard for a Systematic Review (for which many resources already exist). Results: We conducted a literature search and found relevant documents to include in our "search steps" document. This document covers the various search methods included in the day-to-day searches completed by health librarians, and the steps or search moves associated with each of them. Discussion: The need for standardization of practice in health services libraries is especially important as we are surrounded by professions which are accredited and must provide their services within a certain set of standards. The creation of standards is one method to maintain the relevance and value of health services libraries within the healthcare system. Standards also enable us to provide a consistent service experience to our users, especially within multi-site services with both physical library and virtual environment settings.

\section{Social Media Use in Medical and Health Professional Education: Role of the Librarian on a Faculty Steering Committee}

\author{
Natalie Clairoux and Rhoda Weiss-Lambrou \\ Biomedical Librarian, Universitè de Montrèal, Montreal, PQ; Professor of Occupational Therapy at the School of \\ Rehabilitation, Faculty of Medicine, Universitè de Montrèal, Montreal, $P Q$
}

Objective: An interprofessional steering committee was created at Universitè de Montrèal's Faculty of Medicine to examine how social media are integrated in medical and health professional education in universities across the globe, and to propose a strategic plan for integrating social media in the Faculty's various curricula. This presentation will summarize the steering committee's work and describe the librarian's contribution. Methods: The Committee's project leader first conducted a literature search on best practices of social media in medical and health professional curricula. A reference website was then created (mse.med.umontreal.ca) to provide easy access to a large number of the articles and resources reviewed. A steering committee was constituted and 11 meetings were held over 
a 9-month period. The Committee comprised 18 members and included assistant deans, academic program directors, professors, communication advisors, undergraduate and graduate students and a librarian. An online survey on social media use by students and professors of the Faculty was conducted, ten pilot projects were put forward and a three-year strategic plan was proposed. Results: A total of 1508 students and 565 professors participated in the survey. Results showed that both groups had a strong interest in learning how social media could be integrated in academic and professional activities. Participants reported concern with risks associated with social media use and expressed the need for a Faculty policy and guidelines. The librarian's contribution to the steering committee included: writing posts on the website's internal blog, assisting in the design of the survey questionnaires and writing the final report's survey results chapter. She also proposed two pilot projects: creating a social media learning portal and an altmetrics workshop. Discussion: Based on the literature review and the survey results, the Committee affirmed the importance of integrating social media in the various study programs of the Faculty of Medicine. Despite the restricted timeline, this interprofessional steering committee was able to carry out its mandate because of the leadership and expertise of each of its members. As the librarian had the most experience with the use of social media in a professional context, her knowledge was instrumental in assisting the project leader in a group mainly composed of social media non-users.

\title{
Research Data: What's Not Worth Keeping?
}

\author{
Janice Kung and Sandy Campbell \\ Academic Librarian Intern, John W. Scott Health Sciences Library, University of Alberta, Edmonton, AB; Liaison \\ Librarian, John W. Scott Health Sciences Library, University of Alberta, Edmonton, AB
}

Background: The rise of university library involvement in data management has presented numerous challenges for academic libraries. For example, while libraries and archives have always had book and journal collection development policies that defined what they would or would not collect, policies for selecting research data for preservation are in their infancy. This study will help librarians make informed decisions on what type of research data are worth keeping. Objective: The objective of this study is to define which health research data have no archival value and should not be preserved. Researchers will be able to identify what type of data are not worth keeping by exploring the research data management (RDM) practices of health sciences researchers at the University of Alberta (UofA). Methods: A qualitative approach rooted in grounded theory will be used. A survey was sent electronically to UofA's health sciences community. In the next phase of the project, the researchers will followup with semi-structured interviews with survey respondents who express interest in providing additional information on their RDM practices. Results/Discussion: Preliminary findings from the survey indicate that $35 \%$ of researchers have permanently preserved data either in institutional repositories, personal servers, or supplementary material in publications. $41 \%$ of researchers cannot publish data due to confidentiality or proprietary concerns. $65 \%$ of respondents think that some data should not be preserved permanently such as pilot data and un-validated data. Thus, there is a need to develop guidelines to assist researchers with what type of data they should consider keeping.

\section{Looking for Indigenous Health Information: Databases, Hedges, \& International Options}

\author{
Kathy Murray \\ Head, Alaska Medical Library, Anchorage, AK
}

Purpose: This paper will describe strategies for finding indigenous health information from various resources available in the US, Canada and across the circumpolar north countries. Setting/Participants/Resources: The Alaska Medical Library has worked with the National Library of Medicine to create a website for health information for individuals living in the far north. Work on how to search PubMed to find indigenous health information has been done by librarians in the US and Canada and their discoveries will be shared. Brief Description: While the 
website began as a way to improve access to quality health information for Alaska Natives, it has since grown to include publication and research databases, multimedia, climate change, and traditional healing. Content has expanded beyond Alaska and now covers the circumpolar north. Finding published and gray literature for indigenous peoples is made more difficult by the lack of good indexing terms in PubMed. This talk will describe: databases from New Mexico to Alberta and across the globe to Norway which should be considered when looking for health information for indigenous groups and others living in the far north, hedges used to pull information from PubMed and more recently Google Scholar, and why the Arctic Council should be added to your list of great resources. Future opportunities for librarian involvement with the Arctic Council will be shared. Results/Outcome: The growth of this website from local information to both national and international content makes this a good starting point when looking for health information for individuals living in the circumpolar north.

\title{
Online Health Information Avoidance
}

\author{
Colleen Addison and Luanne Freund \\ Doctoral Candidate, School of Library, Archival and Information Studies, University of British Columbia, Vancouver, \\ BC; Associate Professor at the School of Library, Archival and Information Studies, University of British Columbia, \\ Vancouver, $B C$
}

Introduction: Information avoidance is little studied in health and little considered when health information is created. Researchers posit that people in stressful situations are more likely to avoid information, but little is known about how and why, particularly with regards to Internet health information. Our research project aims to discover how stressful situations affect selections of health information websites. Methods: An experimental user study is underway in which participants are tested for individual differences, presented with hypothetical scenarios designed to induce varying stress levels, and then asked to interact with a selection of health information sources. Anticipated results: This study will be conducted in the spring of 2015 and results will be available at the time of the conference. A preliminary online questionnaire was deployed using Mechanical Turk, in which about $7 \%$ of participants indicated some degree of information avoidance when presented with a stressful medical scenario. Reasons included negative affect ("it would worry me") and reliance upon medical practitioners ("I would trust my medical team"). The study also examined connections between emotional state, likelihood of information seeking, and general health, showing that respondents in poor general health had less positive emotion and were less likely to seek information. Contributions: This study will provide much-needed insight into how people interact with health information in times of negative affect and health crises. Findings will contribute to health care and health literacy fields by expanding understanding of information avoidance, and the extent to which stressful situations and individual differences influence such behaviours.

\section{Student Informed Information Literacy Development: Using Curriculum Mapping to Initiate Instructional Collaboration}

\author{
Laura Cobus-Kuo and Lis Chabot \\ Health Sciences Librarian, Ithaca College, Ithaca, NY; College Librarian, Ithaca College, Ithaca, NY
}

Introduction: This two-part assessment study examines an undergraduate Speech Language Pathology and Audiology (SLPA) program. The researchers assessed SLPA student perspectives on information literacy (IL) skills. A curricular review was also performed and an IL curriculum map was developed to communicate strategies for scaffolding IL instruction. Methods: The one-minute-paper (OMP) was used to gather feedback from fourth-year students attending a library session. The OMP asks two questions: what information is useful for coursework, and what areas are still troublesome. Responses were coded and analyzed qualitatively. Concurrently, twenty syllabi for required 
courses were examined and mapped to specific research skills. The results were presented to faculty and administrators. Results: Fifty-one OMPs were collected over a twoyear period. For the first question there were six concepts that students found helpful with the most common response being search strategies. For the second question ten themes emerged for which students had questions with the most common response being there were no questions. From the course syllabi, relevant research skills were identified and categorized into three themes: search strategy, resource, and assessment. Search strategy development and resource knowledge emerged as foci for the first and second year. Critical assessment of information emerged for the third and fourth year. Discussion: The combined assessment supports that IL should be formally integrated into the SLPA curriculum early and often. Reporting the OMP feedback strengthened faculty buy-in while the IL curriculum map provides a strategy to ensure health professions students receive hierarchical IL skill development across the four-year curriculum.

\title{
Acknowledging Academic Librarians' Contributions in Research Publications
}

\author{
Robin Desmueles, Marlene Dorgan, and Sandy Campbell \\ Cataloguing Librarian, Collections Services, McGill University, Montreal, PQ; Head, John W. Scott Health Sciences \\ Library, University of Alberta, Edmonton, AB; Liaison Librarian, John W. Scott Health Sciences Library, University of \\ Alberta, Edmonton, $A B$
}

Background: This project addresses the relevance and value of acknowledgements as scholarly contributions in health sciences librarianship, with a specific focus on the variability in recognition of the work of health sciences librarians in systematic reviews. While acknowledgements are a valid form of recognition, they do present challenges when being used for salary and promotion purposes. Supervisors may not value acknowledgements, it is sometimes unclear what level of effort an acknowledgement represents, those acknowledged may not know that they have been acknowledged, and acknowledgements are not generally indexed, therefore they are difficult to identify. Objective: In this study we explore librarian supervisors' attitudes towards the recognition of the work of their librarians on systematic reviews, with the intention of developing best practices for co-authorship vs acknowledgement of librarians' contributions. Methods: A survey of the literature will be conducted to determine current standards related to authorship and acknowledgement. Librarian Supervisors will be surveyed to elicit their opinions regarding the value of acknowledgements in librarian tenure and promotion. Results/Discussion: Most of the fourteen supervisors who returned surveys expect that their librarians will be involved in systematic reviews either as instructors or as project participants. With one exception, they value LIS authorship equally, slightly or more than systematic review co-authorship. Supervisors generally agreed on a selection of tasks that a librarian would undertake if co-authoring on a systematic review. These roughly parallel a detailed search process for a systematic review. This study will be a springboard for further discussions across Canada as librarians continue to define their role in systematic reviews.

\section{Value and Impact of User-Initiated Literature Searches on Academic and Clinical Practice: A Follow-up Study}

\author{
Lindsay Alcock and Alison Farrell \\ Head of Public Services, Health Sciences Library, Memorial University of Newfoundland, St. John's, NL; Public \\ Services Librarian, Health Sciences Library, Memorial University of Newfoundland, St. John's, NL
}

Introduction: Memorial's HSL offers a literature search service to faculty, staff, residents, and health care providers. A 2012 value and impact study demonstrated that users place a high value on the service and that it has a significant impact on clinical and academic practice. Given the increase in the number of requested searches the 
authors are replicating the study to determine the value and impact on a wider basis as well as compare two years worth of data. Methods: Using the critical incident method of subject selection, all users who requested literature searches in 2014 were invited to complete a survey regarding their experience with and their perceived value of the service. The survey was designed to elicit general satisfaction with the service, identify the nature of the informational need, determine whether or not the information was utilized clinically or academically, whether or not the information was used in an evidence-based model, what was impacted by the information utilization, and how much value was placed on the impact. Results: Initial results indicate a continued overall high perception of the value and impact particularly with respect to academic pursuits. Further analysis will be presented. Discussion: A paucity of research is available on the health library's impact on academic activities. Aside from adding to the body of evidence related to the clinical impact of library services, the intention of this study is also to determine the value of the service in terms of academic pursuits including curriculum planning, evaluation, assessment, research, and professional development. Results continue to inform the development and prioritization of library services at the Health Sciences Library as both an academic and hospital library as well as provide evidence of our user-determined value to stakeholders.

\title{
Mobile Devices at the Bedside: A Collaborative Research Project
}

\author{
Lori Giles-Smith, Christine Shaw, Andrea Spencer, Ceceile Porter, and Michelle \\ Lobchuk
}

Associate Librarian, Health Sciences Libraries, University of Manitoba, Winnipeg, MB; Acting Head, Sciences and Technology Library, University of Manitoba, Winnipeg, MB; Interdisciplinary Educator, Educational Resources, Grace Hospital, Winnipeg, MB; Continuing Education Instructor for NICU and Ambulatory Care Paediatrics, Saint Boniface Hospital, Winnipeg, MB; Associate Professor, College of Nursing, Faculty of Health Sciences, University of Manitoba, Winnipeg, MB

Objectives: Mobile devices are moving library services onto wards and allowing librarians to collaborate with healthcare colleagues in new ways. Librarians are encouraging mobile application (app) use among nurses by purchasing licenses and promoting their potential usefulness at the bedside. However, the extent to which nurses use mobile devices for patient-centred care is unknown. The first objective of this mixed-methods study was to describe current attitudes towards and use of mobile devices/apps among nurses in Winnipeg, MB. The second objective tested the impact of an educational intervention on mobile device/app usage, attitudes and behavior among nurses. Methods: Phase I consisted of a descriptive, cross-sectional survey of in-patient nurses to determine mobile device/app use and attitudes. Phase II involved a one-group pre/post-test design to examine the impact of education sessions led by librarians and hospital educators on nurse attitudes, usage and behaviours. A post-intervention focus group captured thoughts on using mobile devices/apps at the bedside. Results: The study's findings show few nurses are using mobile devices for bedside care though there is strong interest in doing so. Nurses expressed the types of information they need to support bedside care and barriers they encountered using mobile devices/apps in patient-centred care. Discussion: Results indicate nurses are interested in using mobile devices at the bedside and believe there are potential benefits. Librarians and hospital educators should work together to provide the necessary education and support. Larger studies are needed to determine the impact of educational sessions on patient and health provider satisfaction with mobile device use. 


\title{
Assessment of Knowledge and Skills in Allied Health Student Information Literacy Instruction: a Systematic Review
}

\author{
Jill Boruff and Pamela Harrison \\ Liaison Librarian, Life Sciences Library, McGill University, Montreal, PQ; Liaison Librarian, Life Sciences Library, \\ McGill University, Montreal, $P Q$
}

Objectives: This systematic review has two aims. Firstly, our review will determine the type of instructional modalities being implemented, the assessment tools being used, and the knowledge and skills being assessed in information literacy instruction of allied health students. Secondly, we will examine whether existing assessment tools can determine the effectiveness of information literacy instruction in this population. This presentation will explore our methodology and the challenges and patterns that have emerged during our screening process. Methods: The authors are conducting a systematic review of the health science and information science literature to find the methods of information literacy instruction and assessment being used in physical therapy, occupational therapy, speech-language pathology, and nursing. The review will focus on students in academic programs who have been taught information literacy knowledge and skills in either an evidence-based practice context or a stand-alone library session. Results: We will discuss the formulation of the search strategy, the scope of the review, and lessons learned during the screening process. We will also explore trends that have emerged to date, such as the distribution of reported information literacy interventions in individual allied health disciplines, among degree levels, and librarian involvement in instruction. We will not have completed data extraction at this point; we will, however, identify the outcomes that our project explores, including the types of instructional modalities used, the knowledge and skills being assessed, and assessment tools implemented during or following instruction. Discussion: The alignment of learning objectives, instruction modalities and assessment methods is important for effective information literacy instruction. An analysis of current approaches in allied health will demonstrate the extent to which teaching practices in this field evolve in response to evidence derived from assessment, and to discover best practices in building more effective instruction and assessment plans.

\section{Health Science Libraries and Writing Centers: An Innovative Partnership}

\author{
Sarah Chodakewitz and Terry Ann Jankowski \\ Assistant Director and Graduate Tutor, Odegaard Writing and Research Center, University of Washington, Seattle, \\ WA; Assistant Director for User Experience, Health Sciences Library, University of Washington, Seattle, WA
}

Purpose: To illustrate a case study highlighting how provision of writing center peer-tutoring services can successfully expand health science library practice. Setting/ Participants/Resources: This academic health science library provides services for six schools of health sciences, three teaching hospitals, and a variety of research programs. Brief Description: Writing centers that utilize peertutoring strategies have been recognized as an ideal method for assisting writers in developing their communication skills; therefore, many academic libraries have adopted writing centers as partner service providers. However, these centers often orient towards the needs of writers in the humanities and social sciences, ignoring those of students and faculty in the health sciences. This tendency presents a problem, as writing is essential to successful scholarship in health science fields. To address this concern, this library recently initiated a partnership with a campus interdisciplinary writing center to provide peer-tutoring services within the health science library. We hypothesize that by providing services at a location easily accessible to health science writers, and conducted by tutors also pursuing health science degrees, we would address this 
discrepancy. We began by providing a small pilot drop-in center in Autumn 2014; preliminary successes have prompted program expansion. Results: By June 2015, we will have operated this program for one academic year and will be able to provide information to other libraries regarding implementation strategies, user response, and lessons learned. Evaluation: This program will be evaluated based on total sessions offered, utilization of sessions, diversity of fields and users served, and feedback surveys.

\title{
Where Do We Grow From Here? An Evaluation of Library Services Provided to a Provincial Health Authority
}

\author{
Ada Ducas, Sherri Vokey, Tania Gottschalk, and Michael Moffatt \\ Head, Health Sciences Libraries, University of Manitoba, Winnipeg, MB; Head, Neil John Maclean Health Sciences \\ Library, University of Manitoba, Winnipeg, MB; Education Services Librarian, University of Manitoba, Winnipeg, MB; \\ Professor, Community Health Sciences Department and the Pediatrics and Child Health Department, College of \\ Medicine, University of Manitoba, Winnipeg, MB
}

Introduction: Across Canada various models have evolved for providing health sciences libraries services and resources regionally, provincially, and nationally. Equitable access to high-quality evidence-based information for all health science professionals is a key driver. Description: Since 2000, one academic health library and its seven affiliated hospital libraries have provided access to resources and services to over 28,000 staff in a provincial regional health authority. Services include literature searches, document delivery, training, and, for some locations, access to databases, point-of-care tools, and online journals. Our participation in a 2013 large-scale, multi-site study on the value and impact of library and information services on patient care provided critical evaluation data from physicians, residents and nurses.
However, other health professionals were excluded from the study and it did not explore the specifics of the library services provided to this region. Evaluation Method: In 2013 a 24-item web-based survey was created with input from the regional staff, university faculty, and librarians. Survey questions included rating scales and open-ended questions. The survey was distributed by email and received 1000 responses from a diverse group of health professionals. Discussion: The results of this survey provided important information on which libraries, services and resources were used most frequently; how they were rated; and barriers that staff encountered in accessing and using them. Utilizing this information is critical to planning for the evolution and growth of library services within the region.

\section{Radical Collaboration: Medical Librarians, Student Scholarship Competencies and Academic Learning Communities in the 21st Century}

\author{
Rebecca Raworth and Lindsay Alcock \\ Island Medical Program Librarian, University of Victoria, Victoria, BC; Head of Public Services, Health Sciences \\ Library, Memorial University of Newfoundland, St. John's, NL
}

Introduction: CANMEDS roles emphasize the importance of student scholarship and international librarian associations are calling for the need for an embedded model for academic librarians through which librarians may be more engaged with student scholarship. This study was undertaken to determine the extent of librarian involvement in undergraduate medical program student learning communities and in facilitating student achievement of scholarship and research competencies in AAMCaccredited Canadian and American medical schools. Methods: Literature searches were conducted on the topics of renewing undergraduate curricula in relation to 
21 st century educational paradigms, research and scholarship opportunities available to medical students and the changing roles of medical librarians. Two web-based surveys, using both closed and open-ended questions, were developed and distributed: one for Deans, the other for librarians. Both surveys focus on changes occurring as medical schools update their curricula, particularly in the areas of student learning communities, student research and scholarship learning opportunities, and the role of librarians in student learning communities. Results: Results will determine the extent to which librarians with appointments in AAMC-undergraduate medical schools participate in student learning communities and help students achieve research and scholarship competencies. It is hoped that results also reveal the perception that Deans of Curriculum have on the role of librarians in helping students achieve research and scholarship learning outcomes. Discussion: Student learning communities that focus on scholarship and research provide an exciting new and collaborative role for medical librarians.

\title{
The Value of the Library Value Toolkit
}

\author{
Jeanna Hough, Sheila Lacroix, and Miriam Ticoll \\ Manager, Library Services, Halton Healthcare, Oakville, ON; Library Coordinator, Centre for Addiction and Mental \\ Health, Toronto, ON; Executive Director, Health Science Information Consortium of Toronto, Toronto, ON
}

Introduction: The Library Value Toolkit website was launched in October 2014 in response to libraries' need to identify and communicate their value to stakeholders. The toolkit is a website of resources, tools \& tips. Objectives \& Scope: To assess the value of the toolkit, from October 1 2014-Mar 31 2015, 6 months post-launch. The quantitative usage (including overall website traffic, most popular pages, top links \& documents) will inform what topic/ resources are perceived as most important, areas where support is needed, or the perceived value to stakeholders. Qualitative feedback will also be assessed including voluntary feedback provided post-launch and via survey conducted in April. Methods: The toolkit uses the LibGuides CMS system with a tool that tracks, pages, links and document hits/downloads. Qualitative feedback col- lected since launch will also be assessed including input from a feedback survey sent to the HSICT, CHLA/ABSC and MLA listservs in April. Results: While preliminary results suggest that the toolkit is being utilized and qualitative feedback is positive, extent of use and perceived value of the toolkit are largely unknown as the 6 month post-launch period ends on March 31 2015. The full feedback survey will allow for more in-depth results on the pros, cons and how the site/resources are being used practically by Libraries, if the toolkit has added value to those endeavors, and what might increase its use and value. Discussion: The authors will undertake further evaluation and finalize the results of a 6-month, post launch assessment in the Spring of 2015.

\section{Environmental Scan of Repositories of Clinical Trial Data}

\section{Lee-Anne Ufholz}

Regional Sales Manager, Wolters Kluwer Health, Ottawa, ON

Introduction: Inclusion of Individual Participant Data (IPD) in systematic reviews would increase the reliability of produced evidence. The lack of methodologies and standards for data preparation and of relevant repositories are major gaps preventing the availability of raw data. The objective of this study is to explore essential features and practices of repositories that accept clinical trial data and facilitate their sharing and public disclosure. This environmental scan will inform a development of methods and standards for public disclosure of IPDs.
Methods: Environmental scan of repositories that harvest and enable public disclosure of clinical trial data. A list of headings was developed to capture features of selected repositories. We reviewed the literature, searched catalogues of data repositories, analysed respective websites and conducted interviews. Results: Selected repositories are general, institutional, or topic oriented. We analysed predefined features of these repositories including citability and reuse of data. There are no universal standards of data curation and management across repositories but 
they are open to implement such standards if these are defined. Increasingly repositories host IPDs, but there is a need to develop methods and standards for public disclosure of such data. Discussion: This study was the precursor to the creation of an observatory of ongoing transition in clinical research regarding data sharing, opportunities and barriers, named IMPACT (IMProving Access to Clinical Trials data) Observatory. Observatories or natural experiments assess the impact of an intervention that is out of control of the researcher with the ultimate goal to inform the process.

\title{
Evidence-Based Nursing Practice for Specialty Nurses: Piloting a New Curriculum for Hospital- based Infection Control Nurses
}

\author{
Francesca Frati \\ Patient Information Specialist and Instruction Librarian, Jewish General Hospital Health Sciences Library, \\ Montreal, $P Q$
}

Introduction: EBNP is not just EBP for nurses. Hospitalbased nurses make evidence-based decisions about individual patient-care, but also use the evidence to support the development and implementation of policies \& procedures/ CQI. EBNP has evolved from EBM to include several new stages: cultivating curiosity, integrating evidence to guide implementation, evaluating practice change (CQI), and disseminating the evaluation results. This is not reflected in the literature or most EBNP learning materials. An EBM curriculum developed for hematology residents and integrated into courses for undergraduate medicine students and occupational therapists showed that longitudinal, clinically integrated and case-based instruction is effective for improving EBM skills, attitudes and behaviours across specialties. Objectives: To provide longitudinal clinically integrated case-based instruction that better reflects the implementation/CQI aspects of nursing practice.
Methods: A librarian piloted and assessed a new EBNP curriculum integrating content from previous EBM/P courses and from a Nursing Reference Centre EBNP CE module to a convenience sample of seven infection prevention \& control nurses. Five $1.5 \mathrm{~h}$ workshops using real case scenarios are offered over five weeks. Homework and reading assignments are used to reinforce learning. Results: Nurses will complete evaluations after each workshop and a three month post-test and survey are planned to assess knowledge retention and satisfaction with the curriculum. Initial evaluations suggest that nurses believe their practice will be changed or improved and that new knowledge has been acquired. Discussion: This pilot curriculum, modified to support the development and implementation of policies \& procedures/ CQI will be accredited and offered to other nursing specialties.

\section{User Knows Best: A Hospital eLibrary User Experience (Ux) Study}

\author{
Shawn Hendrikx, Sandra Halliday, and Suzanne Maranda \\ Research and Instructional Librarian, Taylor Library, Western University, London, ON; Health Sciences Librarian, \\ Queen's University, Kingston, ON; Head, Bracken Health Sciences Library, Queen's University, Kingston, ON
}

Introduction: As part of an outreach programme with Bracken Health Sciences Library (BHSL) at Queen's University, Hotel Dieu Hospital (HDH) has access to an eLibrary website customized to their institution's information needs. This eLibrary website migrated to WordPress in 2014 to take advantage of the software's cost (free) and built-in responsive design feature (mobile technology friendly). BHSL seized the opportunity to conduct $\mathrm{Ux}$ testing before launching the new eLibrary website. Methods: In the Ux toolkit developed for the librarian researcher, there is an array of Ux testing methodologies that are inexpensive and quick. Five participants recruited from HDH performed the Ux test, and after answering preliminary questions, participants completed fifteen tasks 
encompassing four scenarios using the think-aloud protocol. Debriefing questions followed. The tasks tested each participant's ability to navigate the eLibrary website and revealed areas requiring improvement. After the recommended changes to the $\mathrm{HDH}$ website were made, the Ux testing process was repeated with four of the original participants and one new participant. Results: The Ux tests at $\mathrm{HDH}$ revealed several issues with the eLibrary, including a significant problem with the navigability of the eBook web pages and issues with library language. The investigators noted these obstacles and discussed a variety of solutions. These highlighted areas were improved, and the Ux tests were repeated at $\mathrm{HDH}$ to demonstrate increased website usability. Discussion: The Ux study highlighted areas that worked well and areas for improvement, and this information allowed us to implement an eLibrary website that will hopefully increase user engagement. In the future, BHSL will continue to perform Ux studies to ensure a quality user experience as iterative changes and improvements are made to the eLibrary website.

\title{
Library Support for a Point-of-Care Research Competition
}

\author{
Yvette Ipsaralexi, Chantalle Jack, and Shannon Long \\ Vancouver Community, Vancouver Coastal Health, Vancouver, BC; Librarian, Lions Gate Hospital, Vancouver \\ Coastal Health, Vancouver, BC; Librarian, Richmond Hospital, Vancouver Coastal Health, Richmond, BC
}

Introduction: Each year our health region holds a funding competition for teams of front-line care providers wishing to carry out small clinical research projects. In previous years library support for the teams was encouraged but not mandatory. In 2014, however, it became a requirement that those wanting to participate in the "Research Challenge" attend a library-based literature search training session. Description: Library staff developed a one-hour training workshop that was delivered to each Research Challenge team individually. The goals of the training were to increase familiarity with library research databases, impart knowledge and skills necessary to do basic searches, find literature specifically related to their Research Challenge topics, and be able to evaluate and critique search results. Outcomes: Two months after the training sessions, and once all research proposals were submitted, a follow-up survey was sent to each workshop attendee. The survey inquired about the perceived value of the literature search training as a mandatory component of the Research Challenge and whether attendees applied what they had learned when developing their research proposals. Discussion: The response rate to the follow-up survey was relatively low and we could not determine if a team's success or failure to receive research funding influenced their perception of the usefulness and relevance of the library training. Thus, when determining the role of the library in future research competitions we must use caution in applying these survey results. Regardless, feedback was still valuable in assessing workshop effectiveness and promoting use of the library's services and resources.

\section{"When I Grow Up I Want to Be a Doctor": Promoting Atikamekw Children's Interest for Health Professions Through a Book Collection}

\author{
Monique Clar, Eric Drouin, Bianca Seminaro, and Maryse Fagnant \\ Biomedical Librarian, Universitè de Montrèal, Montreal, PQ; Paediatric Gastroenterologist and Director, Paediatric \\ Gastroenterology, Hepatology and Nutrition Program, CHU Sainte-Justine, Facultè de mèdecine, Universitè de \\ Montrèal, Montreal, PQ; Student, Ecole de bibliothèconomie et des sciences de l'information, Universitè de \\ Montrèal, Montreal, PQ; Student, Facultè de mèdecine, Universitè de Montrèal, Montreal, $P Q$
}

Introduction: Aboriginal peoples are underrepresented within the healthcare professions, and recruitment of Aboriginal students has become a priority for medical schools in Canada. Because of very low high-school completion rates among youth living on-reserve, the Universitè de Montrèal's Faculty of Medicine launched 
in 2011 the Mini-ècole de la santè, a program where health sciences students visit aboriginal schools. Through activities and games, students introduce children to the discovery of health professions. In 2014, the Health Library joined the project with the development of a science books collection for the school libraries and by having a librarian participate in the school visits. Description: In collaboration with the two Atikamekw elementary schools to be visited in 2014, 70 children's books on science, human anatomy and the health professions were selected and purchased for each school by the Health Library. A librarian joined the health sciences students during the school visits and the book collection was integrated in the activities organised during the day. The books were afterwards donated to the school library. Outcomes: Children, school teachers and administrators greatly appreciated the collection. The books were integrated in the library school collections or in the classroom collections. Discussion: Quality school libraries play an important role in student learning, and access to science and health sciences books could enhance children's interest in the health professions. By participating in this project, the library is supporting the Health sciences faculties in achieving their goal of reaching out to Aboriginal children and making them aware that a career in health sciences is possible for them. The collaboration has been successful and will be pursued: the Health library will work with the high schools in the same Atikamekw communities to develop science book collections and the schools will be visited in 2015. A Masters in Library and Information Science student will be joining the Mini-ècole. Upgrading all donated collections is planned as well. 